\title{
$\triangle$-RELATED FUNCTIONS AND GENERALIZED INVERSE LIMITS
}

\author{
TINA SOVIČ \\ University of Maribor, Slovenia
}

\begin{abstract}
For any continuous single-valued functions $f, g:[0,1] \rightarrow$ $[0,1]$ we define upper semicontinuous set-valued functions $F, G:[0,1] \multimap$ $[0,1]$ by their graphs as the unions of the diagonal $\Delta$ and the graphs of setvalued inverses of $f$ and $g$ respectively. We introduce when two functions are $\Delta$-related and show that if $f$ and $g$ are $\Delta$-related, then the inverse $\operatorname{limits} \lim F$ and $\lim G$ are homeomorphic. We also give conditions under which $\underset{0 \_}{\lim } G$ is a quotient space of $\lim F$.
\end{abstract}

\section{INTRODUCTION}

Given two inverse $\operatorname{limits} \lim F$ and $\lim G$, it is usually a very difficult problem to see whether $\lim \stackrel{\circ}{F}$ and $\lim \stackrel{\circ}{G}$ are homeomorphic. That is why there are many authors researching the properties of bonding functions $F$ and $G$ that guarantee the existence of a homeomorphism from $\lim _{0} F$ to $\lim _{0} G$; for examples see $[3,4,5,6,7]$. In present paper we give sufficient conditions on set-valued functions $F$ and $G$ from a large class of upper semicontinuous functions such that their inverse limits are homeomorphic.

Our motivation in defining this class of upper semicontinuous functions is Ingram's paper [8], where the inverse limits with upper semicontinuous functions whose graphs are unions of graphs of single-valued functions are studied. In particular, we start with any continuous function $f:[0,1] \rightarrow[0,1]$ and the identity function $i d:[0,1] \rightarrow[0,1]$, and define the upper semicontinuous

2010 Mathematics Subject Classification. 54F15, 54C60.

Key words and phrases. Inverse limits, upper semicontinuous functions, quotient maps. 
function $F:[0,1] \rightarrow[0,1]$ by

$$
\Gamma(F)=\{(s, t) \in[0,1] \times[0,1] \mid(t, s) \in \Gamma(f) \cup \Gamma(i d)\} .
$$

Our main result says that if $f$ and $g$ are $\Delta$-related, then the inverse limits $\lim F$ and $\lim G$ (where $F$ and $G$ are defined as above) are homeomorphic. We also give conditions under which $\lim G$ is a quotient space of $\lim _{0} F$.

We proceed as follows. In Section 2, the basic definitions and notation are given. Section 3 serves as an illustrative motivation for our results and in Section 4, our main results are presented.

\section{Definitions And notation}

In the paper $\mathbb{N}$ denotes the set of all positive integers and $\mathbb{N}_{0}$ the set of all nonnegative integers. A continuum is a nonempty compact and connected metric space.

For each $\mathbf{x}=\left(x_{1}, x_{2}, x_{3}, \ldots\right)$ in the Hilbert cube $Q=\Pi_{i=1}^{\infty}[0,1]$ we use the standard notation for the $i$-th projection, i.e. $\pi_{i}(\mathbf{x})=x_{i}$. We always use $Q$ to denote the Hilbert cube $\Pi_{i=1}^{\infty}[0,1]$.

$2^{[0,1]}$ denotes the set of all nonempty closed subsets of $[0,1]$. A function $F:[0,1] \rightarrow 2^{[0,1]}$ is called a set-valued function from $[0,1]$ to $[0,1]$. We use $F:[0,1] \multimap[0,1]$ to denote such functions.

A function $F:[0,1] \multimap[0,1]$ is upper semicontinuous at the point $x \in[0,1]$ provided that if $V$ is any open set in $[0,1]$ containing $F(x)$ then there is an open set $U$ in $[0,1]$ containing $x$ such that $F(t) \subseteq V$ for any $t \in U$. A function $F$ is called upper semicontinuous if it is upper semicontinuous at each point of $[0,1]$.

The graph $\Gamma(F)$ of a function $F:[0,1] \multimap[0,1]$ is the set of all points $(x, y) \in[0,1] \times[0,1]$ such that $y \in F(x)$.

The following theorem is a well-known characterization of upper semicontinuous functions ([2, Theorem 1.2]).

Theorem 2.1. Let $F:[0,1] \multimap[0,1]$ be a function. Then $F$ is upper semicontinuous if and only if its graph $\Gamma(F)$ is closed in $[0,1] \times[0,1]$.

In this paper we always deal with inverse sequences $\left\{X_{i}, F_{i}\right\}_{i=1}^{\infty}$, where $X_{i}=[0,1]$ and $F_{i}:[0,1] \multimap[0,1]$ is upper semicontinuous function for each $i$. We denote them by $\left\{[0,1], F_{i}\right\}_{i=1}^{\infty}$. The functions $F_{i}$ are called the bonding functions.

The inverse limit of an inverse sequence $\left\{[0,1], F_{i}\right\}_{i=1}^{\infty}$ is defined to be the subspace of the product space $\prod_{i=1}^{\infty}[0,1]$ of all $\mathbf{x}=\left(x_{1}, x_{2}, x_{3}, \ldots\right) \in$ $\prod_{i=1}^{\infty}[0,1]$, such that $x_{i} \in F_{i}\left(x_{i+1}\right)$ for each $i$. The inverse limit is denoted by $\lim \left\{[0,1], F_{i}\right\}_{i=1}^{\infty}$. These inverse limits are a recent generalization (introduced by T. W. Ingram and W. S. Mahavier) of inverse limits of inverse 
sequences $\left\{[0,1], f_{i}\right\}_{i=1}^{\infty}$, where $f_{i}:[0,1] \rightarrow[0,1]$ are continuous functions. Such inverse limits are usually denoted by $\lim _{\longleftarrow}\left\{[0,1], f_{i}\right\}_{i=1}^{\infty}$. Obviously, for any inverse sequence $\left\{[0,1], f_{i}\right\}_{i=1}^{\infty}$ of compact metric spaces and continuous bonding functions,

$$
\lim _{\longleftarrow}\left\{[0,1], f_{i}\right\}_{i=1}^{\infty}=\lim _{0}\left\{[0,1], F_{i}\right\}_{i=1}^{\infty}
$$

if $F_{i}(x)=\left\{f_{i}(x)\right\}$ for each $i$ and each $x \in[0,1]$.

In the article we deal only with inverse sequences $\left\{[0,1], F_{i}\right\}_{i=1}^{\infty}$ where all the bonding functions are the same. In the case where $F_{i}=F$ for each $i$, the inverse $\operatorname{limit} \lim \left\{[0,1], F_{i}\right\}_{i=1}^{\infty}$ will be denoted by $\lim F$.

Next we introduce some notation that is used in the paper.

For each $t \in[0,1]$ let $\bar{t}=(t, t, t, \ldots)$. Next, let $\Delta=\{(t, t) \mid t \in[0,1]\}$ and

$$
L^{\infty}=\{\bar{t} \mid t \in[0,1]\} .
$$

For any continuous function $f:[0,1] \rightarrow[0,1]$ we define

$$
f^{*}=\{(f(x), x) \mid x \in[0,1]\},
$$

$L_{\left(n_{i}\right)_{i=0}^{k}}(f)=\left\{(\underbrace{t, \ldots, t}_{n_{0}}, \underbrace{f(t), \ldots, f(t)}_{n_{1}}, \ldots, \underbrace{f^{k}(t), \ldots, f^{k}(t)}_{n_{k}}, \overline{f^{k+1}(t)}) \mid t \in[0,1]\right\}$

for each $k \in \mathbb{N}_{0}$ and for any $(k+1)$-tuple $\left(n_{0}, n_{1}, n_{2}, \ldots, n_{k}\right) \in \mathbb{N}^{k+1}$, and

$$
L_{\left(n_{i}\right)_{i=0}^{\infty}}(f)=\left\{(\underbrace{t, \ldots, t}_{n_{0}}, \underbrace{f(t), \ldots, f(t)}_{n_{1}}, \underbrace{f^{2}(t), \ldots, f^{2}(t)}_{n_{2}}, \ldots) \mid t \in[0,1]\right\}
$$

for any sequence $\left(n_{0}, n_{1}, n_{2}, \ldots\right)$ of positive integers.

Next, for each $n_{0} \in \mathbb{N}$ we denote

$$
\begin{aligned}
\mathcal{L}^{n_{0}}(f)= & \left\{L_{\left(n_{i}\right)_{i=0}^{k}}(f) \mid k \in \mathbb{N}_{0} \text { and } n_{1}, n_{2}, \ldots, n_{k} \in \mathbb{N}\right\} \\
& \cup\left\{L_{\left(n_{i}\right)_{i=0}^{\infty}}(f) \mid n_{i} \in \mathbb{N} \text { for each } i \in \mathbb{N}\right\},
\end{aligned}
$$

and

$$
L^{n_{0}}(f)=\bigcup \mathcal{L}^{n_{0}}(f)
$$

meaning that $L^{n_{0}}(f)$ is the union of sets from $\mathcal{L}^{n_{0}}(f)$.

\section{Motivation examples}

In this section we take three simple u.s.c. functions from $[0,1]$ to $[0,1]$ and study relationships of their inverse limits. Those functions will serve as a motivation for our main results.

We define each of the three functions by defining their graphs. The graph of each function is the union of the diagonal $\Delta$ and the set $f^{*}$ for some continuous function $f:[0,1] \rightarrow[0,1]$. We define the three functions in such a 
way that their inverse limits are not homeomorphic, but still, they are related in the sense that there is a quotient map from one to another. First we prove the following proposition.

Proposition 3.1. Let $f:[0,1] \rightarrow[0,1]$ be a continuous function and $F:[0,1] \multimap[0,1]$ the upper semicontinuous function defined by $\Gamma(F)=\Delta \cup f^{*}$. Then

$$
\lim _{\circ} F=\mathrm{Cl}\left(\bigcup_{n_{0} \in \mathbb{N}} L^{n_{0}}(f)\right)=\left(\bigcup_{n_{0} \in \mathbb{N}} L^{n_{0}}(f)\right) \cup L^{\infty} .
$$

Proof. The equalities

$$
\lim _{\circ} F=\left(\bigcup_{n_{0} \in \mathbb{N}} L^{n_{0}}(f)\right) \cup L^{\infty}
$$

and

$$
\mathrm{Cl}\left(\bigcup_{n_{0} \in \mathbb{N}} L^{n_{0}}(f)\right)=\left(\bigcup_{n_{0} \in \mathbb{N}} L^{n_{0}}(f)\right) \cup L^{\infty}
$$

are obvious. We leave the details to a reader.

EXAMPle 3.2. Let $f:[0,1] \rightarrow[0,1]$ be the piecewise linear function, whose graph is the union of two straight line segments connecting the points $(0,1),\left(\frac{1}{2}, \frac{3}{4}\right)$ and $(1,1)$. We define $F:[0,1] \multimap[0,1]$ by $\Gamma(F)=\Delta \cup f^{*}$. See Figure 1 .
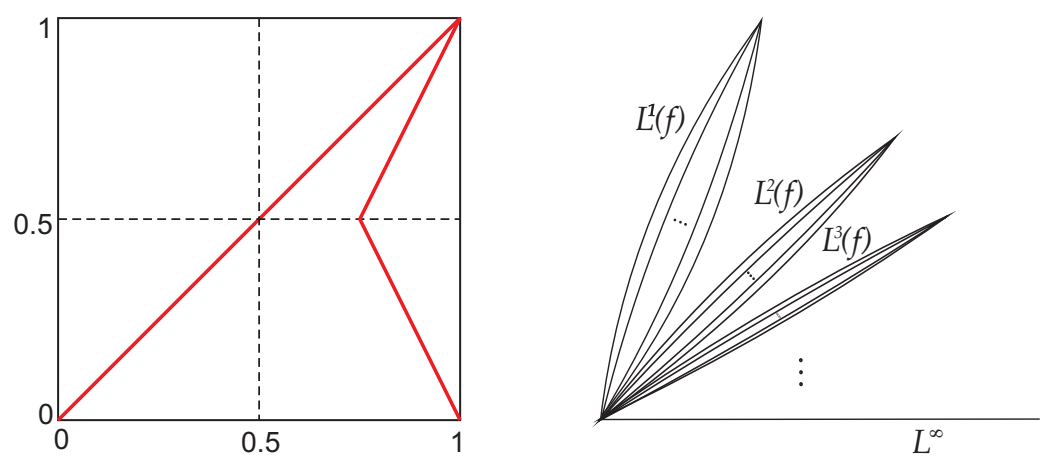

Figure 1. $\Gamma(F)$ (left) and a homeomorphic copy of $\lim F$ (right)

Then by Proposition 3.1,

$$
\lim _{\circ} F=\mathrm{Cl}\left(\bigcup_{n_{0} \in \mathbb{N}} L^{n_{0}}(f)\right)=\left(\bigcup_{n_{0} \in \mathbb{N}} L^{n_{0}}(f)\right) \cup L^{\infty} .
$$


For a geometrical interpretation of the inverse limit, let $n_{0}$ be arbitrarily chosen. One can easily see that each element of $\mathcal{L}^{n_{0}}(f)$ is an arc with endpoints $(\underbrace{0,0, \ldots, 0}_{n_{0}}, \overline{1})$ and $\overline{1}$, and that $L^{n_{0}}(f) \cap\left(\left\{t_{0}\right\} \times Q\right)$ is a Cantor set for each $t_{0} \in(0,1)$.

Also, note that $L^{n_{0}}(f) \cap L^{n_{0}^{\prime}}(f)=\{\overline{1}\}$ if and only if $n_{0} \neq n_{0}^{\prime}, L^{n_{0}}(f) \cap$ $L^{\infty}=\{\overline{1}\}$ for each $n_{0} \in \mathbb{N}$ and $\lim _{n_{0} \rightarrow \infty} L^{n_{0}}(f)=L^{\infty}$; see Figure 1.

Next we define the second function of the example.

Let $g:[0,1] \rightarrow[0,1]$ be the piecewise linear function, whose graph is the union of four straight line segments connecting the points $(0,1),\left(\frac{1}{4}, \frac{7}{8}\right),\left(\frac{1}{2}, 1\right)$, $\left(\frac{3}{4}, \frac{7}{8}\right)$ and $(1,1)$.

We define $G:[0,1] \multimap[0,1]$ by $\Gamma(G)=\Delta \cup g^{*}$. See Figure 2 .
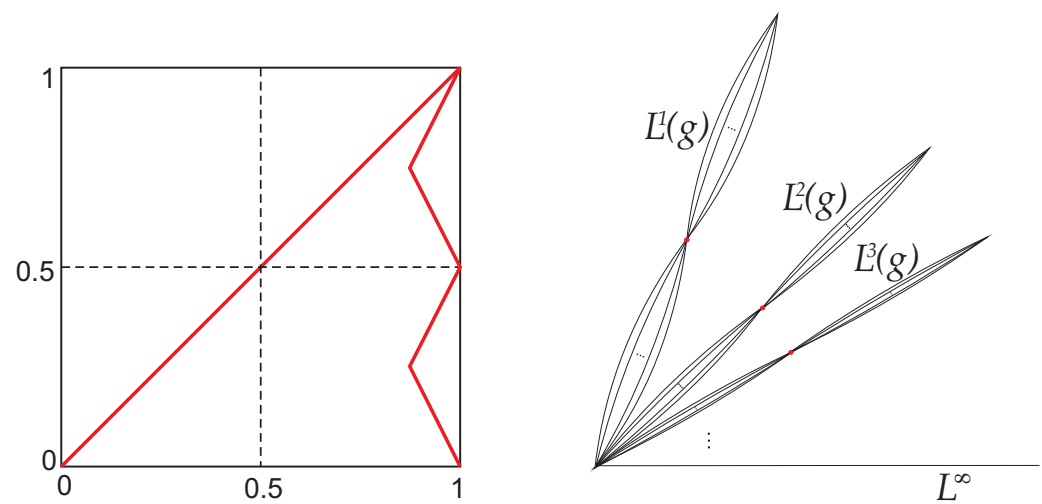

Figure 2. $\Gamma(G)$ (left) and a homeomorphic copy of $\lim G$ (right)

Then by Proposition 3.1,

$$
\lim _{\circ} G=\mathrm{Cl}\left(\bigcup_{n_{0} \in \mathbb{N}} L^{n_{0}}(g)\right)=\left(\bigcup_{n_{0} \in \mathbb{N}} L^{n_{0}}(g)\right) \cup L^{\infty} .
$$

Let $\varphi: \lim _{0} F \rightarrow \lim _{0} G$ be defined by

$$
\begin{gathered}
\varphi(\underbrace{t, t, \ldots, t}_{n_{0}}, \underbrace{f(t), f(t), \ldots, f(t)}_{n_{1}}, \underbrace{f^{2}(t), f^{2}(t), \ldots, f^{2}(t)}_{n_{2}}, \ldots) \\
=(\underbrace{t, t, \ldots, t}_{n_{0}}, \underbrace{g(t), g(t), \ldots, g(t)}_{n_{1}}, \underbrace{g^{2}(t), g^{2}(t), \ldots, g^{2}(t)}_{n_{2}}, \ldots) .
\end{gathered}
$$


It is easy to see that $\varphi$ is well defined and surjective. Since $g$ is continuous, $\varphi$ is also a continuous function. This means that $\varphi$ is a quotient map from $\lim F$ to the $\lim G$.

Note that $\varphi$ is not injective. For instance, let

$$
\mathbf{x}_{n}=(\frac{1}{2}, \underbrace{f\left(\frac{1}{2}\right), f\left(\frac{1}{2}\right), \ldots, f\left(\frac{1}{2}\right)}_{n}, \overline{f^{2}\left(\frac{1}{2}\right)}) \in L_{(1, n)}(f)
$$

for each positive integer $n$. Recall that $L_{(1, n)}(f)$ are $\operatorname{arcs}$ with

$$
\bigcap_{n \in \mathbb{N}} L_{(1, n)}(f)=\{(0, \overline{1}), \overline{1}\}
$$

and therefore $\mathbf{x}_{n} \neq \mathbf{x}_{m}$ for each $n \neq m$. But obviously $\varphi\left(\mathbf{x}_{n}\right)=\left(\frac{1}{2}, \overline{1}\right)$ for each positive integer $n$. See Figure 2, where $\lim G$ is presented.

Finally, the last function of the example is defined.

Let $h:[0,1] \rightarrow[0,1]$ be the piecewise linear function, whose graph is the union of four straight line segments connecting the points $(0,1),\left(\frac{1}{4}, \frac{3}{8}\right),\left(\frac{1}{2}, 1\right)$, $\left(\frac{3}{4}, \frac{7}{8}\right)$ and $(1,1)$.

We define $H:[0,1] \multimap[0,1]$ by $\Gamma(H)=\Delta \cup h^{*}$. See Figure 3 .

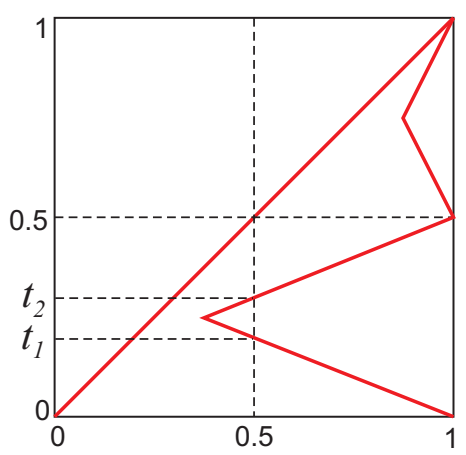

Figure 3. $\Gamma(H)$

Then by Proposition 3.1,

$$
\underset{\sim}{\lim } H=\mathrm{Cl}\left(\bigcup_{n_{0} \in \mathbb{N}} L^{n_{0}}(h)\right)=\left(\bigcup_{n_{0} \in \mathbb{N}} L^{n_{0}}(h)\right) \cup L^{\infty} .
$$


As before, one can easily see that

$$
\begin{gathered}
\psi(\underbrace{t, t, \ldots, t}_{n_{0}}, \underbrace{g(t), g(t), \ldots, g(t)}_{n_{1}}, \underbrace{g^{2}(t), g^{2}(t), \ldots, g^{2}(t)}_{n_{2}}, \ldots) \\
=(\underbrace{t, t, \ldots, t}_{n_{0}}, \underbrace{h(t), h(t), \ldots, h(t)}_{n_{1}}, \underbrace{h^{2}(t), h^{2}(t), \ldots, h^{2}(t)}_{n_{2}}, \ldots)
\end{gathered}
$$

defines a quotient map $\lim _{0} G \rightarrow \underset{0}{\lim } H$.

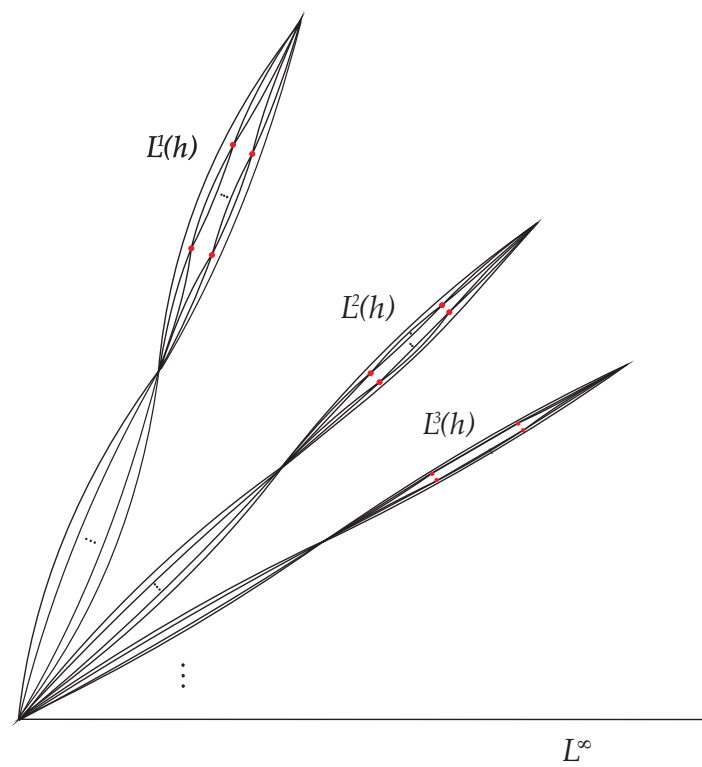

FiguRe 4. A homeomorphic copy of $\lim H$

We show that $\psi$ is not injective. From the definition of the function $h$ it follows that there exist $t_{1}, t_{2} \in[0,1]$ with $t_{1} \neq t_{2}$ and $h\left(t_{1}\right)=h\left(t_{2}\right)=\frac{1}{2}$. Let

$$
\mathbf{x}_{n}=(t_{1}, g\left(t_{1}\right), \underbrace{g^{2}\left(t_{1}\right), g^{2}\left(t_{1}\right), \ldots, g^{2}\left(t_{1}\right)}_{n}, \overline{g^{3}\left(t_{1}\right)})
$$

and

$$
\mathbf{y}_{n}=(t_{2}, g\left(t_{2}\right), \underbrace{g^{2}\left(t_{2}\right), g^{2}\left(t_{2}\right), \ldots, g^{2}\left(t_{2}\right)}_{n}, \overline{g^{3}\left(t_{2}\right)}) \text {. }
$$


Since $\mathbf{x}_{n}, \mathbf{y}_{n} \in L_{(1,1, n)}(g)$ and

$$
\bigcap_{n \in \mathbb{N}} L_{(1,1, n)}(g)=\{(0,0, \overline{1}), \overline{1}\},
$$

it holds that $\mathbf{x}_{n} \neq \mathbf{x}_{m}$ and $\mathbf{y}_{n} \neq \mathbf{y}_{m}$ for each $n \neq m$. But $\psi\left(\mathbf{x}_{n}\right)=\left(t_{1}, \frac{1}{2}, \overline{1}\right)$ and $\psi\left(\mathbf{y}_{n}\right)=\left(t_{2}, \frac{1}{2}, \overline{1}\right)$ for each positive integer $n$.

See Figure 4, where $\lim H$ is presented. On the Figure some special points of $\lim H$ which are not special for $\lim G$ may be seen; i.e., within the upper loops, sets of ramification points appear in $\lim H$ : one set associated with $t_{1}$ (the ramification points on the left hand part of the upper loops) and the other set associated with $t_{2}$ (the ramification points on the right hand part of the upper loops).

We can define a quotient map $\lim _{0} F \rightarrow \lim _{\circ} H$ by using the same formula as before.

Note that the harmonic fan (which is homeomorphic to the inverse limit $\lim \Lambda$ where $\Gamma(\Lambda)=\Delta \cup(\{1\} \times[0,1])$, see [2, p. 31] for instance) is a quotient space of each inverse limit from the example. To see this, a reader may follow a similar arguing as above.

\section{MAin Results}

In the present section we give our main results. In particular, for continuous functions $f, g:[0,1] \rightarrow[0,1]$ and the upper semicontinuous functions $F, G:[0,1] \multimap[0,1]$, where

$$
\Gamma(F)=\Delta \cup f^{*} \text { and } \Gamma(G)=\Delta \cup g^{*},
$$

we give sufficient conditions on $f$ and $g$ under which inverse limits $\lim F$ and $\lim G$ are homeomorphic. We introduce some notation first.

For a continuous function $f:[0,1] \rightarrow[0,1]$ let

$$
\begin{aligned}
& A_{0}(f)=\{x \in[0,1] \mid f(x)=x\}, \\
& A_{i}(f)=\left\{x \in[0,1] \mid f^{i+1}(x)=f^{i}(x)\right\} \text { for each } i \in \mathbb{N},
\end{aligned}
$$

and let

$$
A(f)=\bigcup_{i=0}^{\infty} A_{i}(f) .
$$

Definition 4.1. Let $f:[0,1] \rightarrow[0,1]$ and $g:[0,1] \rightarrow[0,1]$ be continuous functions. We say that $f$ and $g$ are $\Delta$-related, if there is an increasing homeomorphism $\alpha:[0,1] \rightarrow[0,1]$ such that $\alpha\left(A_{i}(f)\right)=A_{i}(g)$ for each nonnegative integer $i$. 
Note that the condition " $\alpha\left(A_{i}(f)\right)=A_{i}(g)$ for each nonnegative integer $i$ " in Definition 4.1 is equivalent to " $\alpha\left(A_{i+1}(f) \backslash A_{i}(f)\right)=A_{i+1}(g) \backslash A_{i}(g)$ for each nonnegative integer $i$ " as well as to " $\left.\alpha\right|_{A_{i}(f)}: A_{i}(f) \rightarrow A_{i}(g)$ is an increasing homeomorphism for each nonnegative integer $i$ ".

TheOREM 4.2. Let $f, g:[0,1] \rightarrow[0,1]$ be $\Delta$-related continuous functions and let $F, G:[0,1] \multimap[0,1]$ be defined by their graphs:

$$
\Gamma(F)=\Delta \cup f^{*}, \Gamma(G)=\Delta \cup g^{*} .
$$

Then $\lim _{0} F$ is homeomorphic to $\lim _{0} G$.

Proof. Let $\alpha:[0,1] \rightarrow[0,1]$ be an increasing homeomorphism, such that $\alpha\left(A_{i}(f)\right)=A_{i}(g)$ for each nonnegative integer $i$.

We define $\varphi: \lim _{0} F \rightarrow \lim _{0} G$ by

$$
\begin{aligned}
& \varphi(\underbrace{t, \ldots, t}_{n_{0}}, \underbrace{f(t), \ldots, f(t)}_{n_{1}}, \underbrace{f^{2}(t), \ldots, f^{2}(t)}_{n_{2}}, \ldots) \\
& =(\underbrace{\alpha(t), \ldots, \alpha(t)}_{n_{0}}, \underbrace{g(\alpha(t)), \ldots, g(\alpha(t))}_{n_{1}}, \underbrace{g^{2}(\alpha(t)), \ldots, g^{2}(\alpha(t))}_{n_{2}}, \ldots)
\end{aligned}
$$

and show that $\varphi$ is a homeomorphism. Note that $n_{0}>0$.

Obviously, $\varphi$ is well defined and since $\alpha$ and $g$ are both continuous, $\varphi$ is also a continuous function. Since for each

$$
\mathbf{y}=(\underbrace{t, \ldots, t}_{n_{0}}, \underbrace{g(t), \ldots, g(t)}_{n_{1}}, \underbrace{g^{2}(t), \ldots, g^{2}(t)}_{n_{2}}, \ldots) \in \lim _{0} G
$$

there is

$$
\begin{aligned}
& \mathbf{x}=(\underbrace{\alpha^{-1}(t), \ldots, \alpha^{-1}(t)}_{n_{0}}, \underbrace{f\left(\alpha^{-1}(t)\right), \ldots, f\left(\alpha^{-1}(t)\right)}_{n_{1}}, \\
& \underbrace{f^{2}\left(\alpha^{-1}(t)\right), \ldots, f^{2}\left(\alpha^{-1}(t)\right)}_{n_{2}}, \ldots) \in \lim _{\circ} F
\end{aligned}
$$

such that $\varphi(\mathbf{x})=\mathbf{y}$ it follows that $\varphi$ is surjective. To show that $\varphi$ is injective let $\varphi\left(\mathbf{x}_{1}\right)=\varphi\left(\mathbf{x}_{2}\right)$. We already know that there exist $t, s \in[0,1]$ and $n_{i}, m_{i} \in$ $\mathbb{N} \cup\{\infty\}$ for each nonnegative integer $i$ such that

$$
\begin{aligned}
\varphi\left(\mathbf{x}_{1}\right) & =\varphi(\underbrace{t, \ldots, t}_{n_{0}}, \underbrace{f(t), \ldots, f(t)}_{n_{1}}, \ldots) \\
& =(\underbrace{\alpha(t), \ldots, \alpha(t)}_{n_{0}}, \underbrace{g(\alpha(t)), \ldots, g(\alpha(t))}_{n_{1}}, \ldots)
\end{aligned}
$$


and

$$
\begin{aligned}
\varphi\left(\mathbf{x}_{2}\right) & =\varphi(\underbrace{s, \ldots, s}_{m_{0}}, \underbrace{f(s), \ldots, f(s)}_{m_{1}}, \ldots) \\
& =(\underbrace{\alpha(s), \ldots, \alpha(s)}_{m_{0}}, \underbrace{g(\alpha(s)), \ldots, g(\alpha(s))}_{m_{1}}, \ldots) .
\end{aligned}
$$

It follows that $\alpha(t)=\alpha(s)$ and therefore $t=s$ since $\alpha$ is bijective. We have

$$
\begin{aligned}
\varphi\left(\mathbf{x}_{1}\right) & =(\underbrace{\alpha(t), \ldots, \alpha(t)}_{n_{0}}, \underbrace{g(\alpha(t)), \ldots, g(\alpha(t))}_{n_{1}}, \underbrace{g^{2}(\alpha(t)), \ldots, g^{2}(\alpha(t))}_{m_{0}}, \ldots) \\
& =(\underbrace{\alpha(t), \ldots, \alpha(t)}_{n_{2}}, \underbrace{g(\alpha(t)), \ldots, g(\alpha(t))}_{m_{2}}, \underbrace{g^{2}}_{g_{1}(\alpha(t)), \ldots, g^{2}(\alpha(t))}, \ldots) \\
& =\varphi\left(\mathbf{x}_{2}\right) .
\end{aligned}
$$

Suppose that $n_{0} \neq m_{0}$. Then $g(\alpha(t))=\alpha(t)$ and therefore $\alpha(t) \in A_{0}(g)$. It follows that $t \in A_{0}(f)$ and $\mathbf{x}_{1}=\bar{t}=\mathbf{x}_{2}$.

Next, suppose that there exists $k \in \mathbb{N}$ such that $n_{i}=m_{i}$ for each $i<k$ and $n_{k} \neq m_{k}$.

Then $g^{k+1}(\alpha(t))=g^{k}(\alpha(t))$ and therefore $\alpha(t) \in A_{k}(g)$ and $t \in A_{k}(f)$. Thus $\pi_{i}\left(\mathbf{x}_{1}\right)=t=\pi_{i}\left(\mathbf{x}_{2}\right)$ for each $i \geq k$ and since (by the assumption) $\pi_{i}\left(\mathbf{x}_{1}\right)=\pi_{i}\left(\mathbf{x}_{2}\right)$ for each $i<k$, it follows that $\mathbf{x}_{1}=\mathbf{x}_{2}$. Therefore $\varphi$ is a homeomorphism.

Next we interpret the relation "to be $\Delta$-related functions" for the class of continuous functions $f:[0,1] \rightarrow[0,1]$, such that $A(f)$ is finite. This interpretation gives an easy tool to detect $\Delta$-related functions.

Note that if $A(f)$ is finite, then also $A_{i}(f)$ is finite for each $i$ and since $A_{i}(f) \subseteq A_{i+1}(f)$ for each $i$, there is $k \in \mathbb{N}$ such that $A_{i}(f)=A_{i+1}(f)$ for each $i \geq k$.

Theorem 4.3. Let $f, g:[0,1] \rightarrow[0,1]$ be continuous functions such that $A(f)$ and $A(g)$ are finite and let for each $i \in \mathbb{N}_{0}, A_{i}(f)=\left\{a_{1}^{i}, a_{2}^{i}, \ldots, a_{n_{i}}^{i}\right\}$, where $a_{j}^{i}<a_{j+1}^{i}$ for each $j \in\left\{1,2, \ldots, n_{i}-1\right\}$, and $A_{i}(g)=\left\{b_{1}^{i}, b_{2}^{i}, \ldots, b_{m_{i}}^{i}\right\}$, where $b_{j}^{i}<b_{j+1}^{i}$ for each $j \in\left\{1,2, \ldots, m_{i}-1\right\}$. Then $f$ and $g$ are $\Delta$-related if and only if the following hold true

1. $\left|A_{i}(f)\right|=\left|A_{i}(g)\right|$ for each $i \in \mathbb{N}_{0}$,

2. $0 \in A_{i}(f)$ if and only if $0 \in A_{i}(g)$ for each $i \in \mathbb{N}_{0}$,

$1 \in A_{i}(f)$ if and only if $1 \in A_{i}(g)$ for each $i \in \mathbb{N}_{0}$,

3. $\left|A_{i+1}(f) \cap\left[0, a_{j}^{i}\right]\right|=\left|A_{i+1}(g) \cap\left[0, b_{j}^{i}\right]\right|$ for each $j \in\left\{1,2, \ldots, n_{i}\right\}$.

Note that (3) of Theorem 4.3 is equivalent to (3') and (3") below (3') $\left|A_{i+1}(f) \cap\left[a_{j}^{i}, 1\right]\right|=\left|A_{i+1}(g) \cap\left[b_{j}^{i}, 1\right]\right|$ for each $j \in\left\{1,2, \ldots, n_{i}\right\}$. 
(3") $\left|A_{i+1}(f) \cap\left[0, a_{1}^{i}\right]\right|=\left|A_{i+1}(g) \cap\left[0, b_{1}^{i}\right]\right|,\left|A_{i+1}(f) \cap\left[a_{n_{i}}^{i}, 1\right]\right|=\mid A_{i+1}(g) \cap$ $\left[b_{n_{i}}^{i}, 1\right] \mid$ and $\left|A_{i+1}(f) \cap\left[a_{j}^{i}, a_{j+1}^{i}\right]\right|=\left|A_{i+1}(g) \cap\left[b_{j}^{i}, b_{j+1}^{i}\right]\right|$ for each $j \in$ $\left\{1,2, \ldots, n_{i}-1\right\}$.

Proof. First we prove that if $f$ and $g$ are $\Delta$-related, then (1), (2) and (3) follows. Let $\alpha:[0,1] \rightarrow[0,1]$ be an increasing homeomorphism with $\alpha\left(A_{i}(f)\right)=A_{i}(g)$ for each nonnegative integer $i$. Since $0 \leq a_{1}^{i}<a_{2}^{i}<\ldots<$ $a_{n_{i}}^{i} \leq 1$ for each nonnegative integer $i$, it follows that $\alpha(0)=0, \alpha(1)=1$ and $\alpha\left(a_{j}^{i}\right)=b_{j}^{i}$ for each $j \in\left\{1,2, \ldots, n_{i}\right\}$ and therefore (1) and (2) obviously hold true. Suppose that $A_{i+1}(f) \cap\left[0, a_{j}^{i}\right]=\left\{a_{1}^{i+1}, a_{2}^{i+1}, \ldots, a_{k}^{i+1}\right\}$ for some $k \leq n_{i+1}$. Since $\alpha\left(\left[0, a_{j}^{i}\right]\right)=\left[0, b_{j}^{i}\right]$ and $\alpha\left(A_{i+1}(f)\right)=A_{i+1}(g)$ it follows that (note that $\alpha$ is an increasing homeomorphism and $A_{i+1}(f), A_{i+1}(g)$ are finite) $\alpha\left(A_{i+1}(f) \cap\left[0, a_{j}^{i}\right]\right)=A_{i+1}(g) \cap\left[0, b_{j}^{i}\right]$ and therefore (3) follows.

To prove the other implication, suppose that (1), (2) and (3) hold true. Let $\alpha:[0,1] \rightarrow[0,1]$ be the increasing piecewise linear function, such that $\alpha(0)=0, \alpha(1)=1$ and $\alpha\left(a_{j}^{i}\right)=b_{j}^{i}$ for each $i \in \mathbb{N}_{0}$ and each $j \in\left\{1,2, \ldots, n_{i}\right\}$. Obviously $\alpha$ is an increasing homeomorphism with $\alpha\left(A_{i}(f)\right)=A_{i}(g)$ for each nonnegative integer $i$ and therefore $f$ and $g$ are $\Delta$-related.

The following is an easy corollary of Theorem 4.2 and Theorem 4.3.

Corollary 4.4. Let $f, g:[0,1] \rightarrow[0,1]$ be continuous functions such that $A(f)$ and $A(g)$ are finite. Further, suppose that the following hold true

1. $\left|A_{i}(f)\right|=\left|A_{i}(g)\right|$ for each $i \in \mathbb{N}_{0}$,

2. $0 \in A_{i}(f)$ if and only if $0 \in A_{i}(g)$ for each $i \in \mathbb{N}_{0}$, $1 \in A_{i}(f)$ if and only if $1 \in A_{i}(g)$ for each $i \in \mathbb{N}_{0}$,

3. $\left|A_{i+1}(f) \cap\left[0, a_{j}^{i}\right]\right|=\left|A_{i+1}(g) \cap\left[0, b_{j}^{i}\right]\right|$ for each $j \in\left\{1,2, \ldots, n_{i}\right\}$.

Then $\lim _{\mathrm{O}} F$ is homeomorphic to $\lim _{\mathrm{O}} G$.

ExAmple 4.5. Let $F, G:[0,1] \multimap[0,1]$ be defined by their graphs $\Gamma(F)$ and $\Gamma(G)$, as shown on Figure 5. Then by Corollary $4.4 \lim F$ is homeomorphic to $\lim G$.

In Theorem 4.6, conditions on $f$ and $g$ are presented, under which the existence of a quotient map (and not necessarily a homeomorphism) from $\lim _{0} F$ to $\lim _{0} G$ is accomplished.

THEOREM 4.6. Let $f, g:[0,1] \rightarrow[0,1]$ be continuous functions such that $A_{i}(f) \subseteq A_{i}(g)$ for each nonnegative integer $i$. Further, let $F, G:[0,1] \multimap[0,1]$ be defined by $\Gamma(F)=\Delta \cup f^{*}, \Gamma(G)=\Delta \cup g^{*}$. Then $\lim _{\circ} G$ is a quotient space of $\lim F$. 

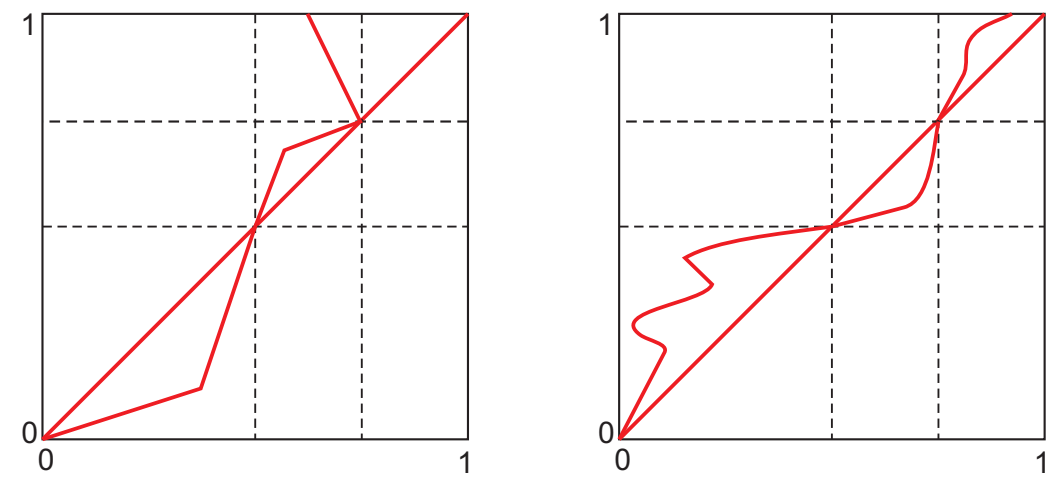

Figure 5. $\Gamma(F)$ (left) and $\Gamma(G)$ (right)

Proof. We define $\varphi: \lim _{0} F \rightarrow \lim _{0} G$ by

$$
\begin{gathered}
\varphi(\underbrace{t, \ldots, t}_{n_{0}}, \underbrace{f(t), \ldots, f(t)}_{n_{1}}, \underbrace{f^{2}(t), \ldots, f^{2}(t)}_{n_{2}}, \ldots) \\
=(\underbrace{t, \ldots, t}_{n_{0}}, \underbrace{g(t), \ldots, g(t)}_{n_{1}}, \underbrace{g^{2}(t), \ldots, g^{2}(t)}_{n_{2}}, \ldots)
\end{gathered}
$$

and show that $\varphi$ is a quotient map. It is easy to see that $\varphi$ is well defined and surjective since $A_{i}(f) \subseteq A_{i}(g)$ for each nonnegative integer $i$. Since $g$ is continuous, $\varphi$ is also a continuous function.

REMARK 4.7. Let $\varphi$ be the function from the proof of Theorem 4.6 and suppose that $A(f)$ and $A(g)$ are finite. The quotient map $\varphi$ helps picturing the inverse limit $\lim _{0} G$ by gluing some points from $\lim _{0 \ldots} F$ together. We continue by listing such points.

We show that $\varphi$ is injective if and only $A_{i}(f)=A_{i}(g)$ for each nonnegative integer $i$. If $A_{i}(f)=A_{i}(g)$ for each $i$, then by taking $\alpha:[0,1] \rightarrow[0,1], \alpha(t)=t$ we can see that $f$ and $g$ are $\Delta$-related and $\varphi$ is a homeomorphism. Suppose 
that there exists $i \in \mathbb{N}_{0}$ such that $A_{i}(f)$ is a proper subset of $A_{i}(g)$ and let

$$
\begin{gathered}
B_{i}(f)=\left\{(\underbrace{t, \ldots, t}_{n_{0}}, \underbrace{f(t), \ldots, f(t)}_{n_{1}}, \ldots, \underbrace{f^{i}(t), \ldots, f^{i}(t)}_{n_{i}}, f^{i+1}(t), \ldots)\right. \\
\left.\mid t \in A_{i}(g) \backslash A_{i}(f)\right\} \subseteq \lim _{\circ} F \\
B_{i}(g)=\left\{(\underbrace{t, \ldots, t}_{n_{0}}, \underbrace{g(t), \ldots, g(t)}_{n_{1}}, \ldots, \underbrace{g^{i}(t), \ldots, g^{i}(t)}_{n_{i}}, g^{i+1}(t), \ldots)\right. \\
\left.\mid t \in A_{i}(g) \backslash A_{i}(f)\right\} \subseteq \lim _{\circ} G .
\end{gathered}
$$

Then $\varphi\left(B_{i}(f)\right)=B_{i}(g)$ and since $f^{i+1}(t) \neq f^{i}(t)$ and $g^{i+1}(t)=g^{i}(t)$ it holds that $\left|B_{i}(f)\right|>\left|B_{i}(g)\right|$, since both, $B_{i}(f)$ and $B_{i}(g)$ are finite. Therefore $\varphi$ is not injective.

We conclude the paper with the following illustrative example.

Example 4.8. Let $F, G:[0,1] \multimap[0,1]$ be defined by their graphs $\Gamma(F)$ and $\Gamma(G)$, as shown on Figure 6 . Then by Theorem $4.6 \lim _{\circ} G$ is a quotient
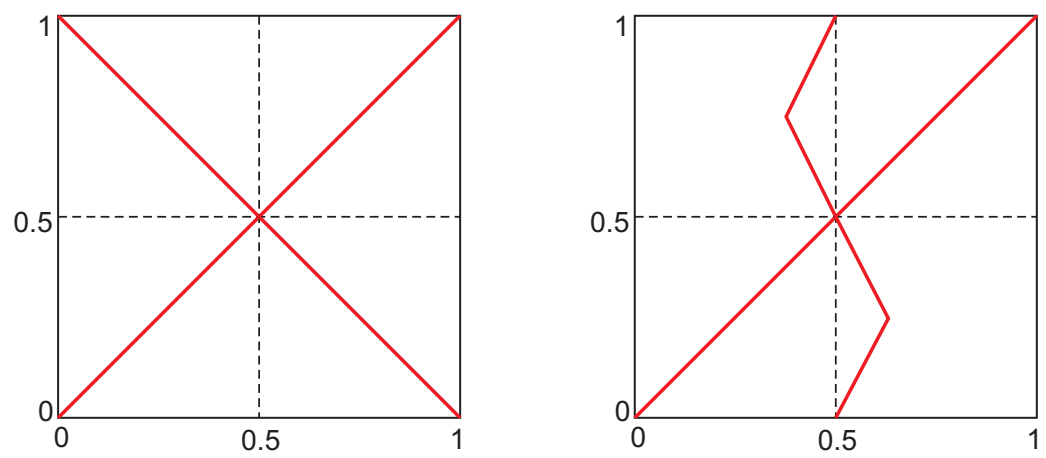

Figure 6. $\Gamma(F)$ (left) and $\Gamma(G)$ (right)

space of $\lim _{0} F$. Note that $\lim _{0} F$ is a Cantor fan (see [2, p. 22]), while a homeomorphic copy of the quotient space $\lim _{0 \_} G$ is seen on Figure 7 .

\section{ACKNOWLEDGEMENTS.}

The author thanks Iztok Banič and Uroš Milutinović for helpful suggestions and discussions. The author also thanks the anonymous referees for useful remarks. 


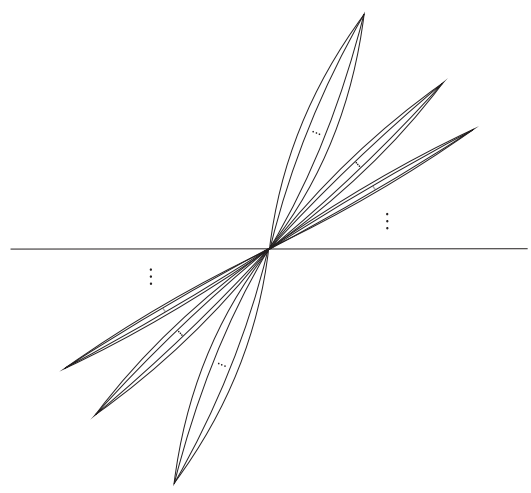

FiguRE 7. A homeomorphic copy of $\lim G$ from Example 4.8

\section{REFERENCES}

[1] A. Illanes and S. B. Nadler, Hyperspaces. Fundamentals and recent advances, Marcel Dekker, Inc., New York, 1999.

[2] W. T. Ingram, An introduction to inverse limits with set-valued functions, Springer, New York, 2012.

[3] S. E. Holte, Inverse limits of Markov interval maps, Topology Appl. 123 (2002), 421-427.

[4] I. Banič and T. Lunder, Inverse limits with generalized Markov interval functions, Bull. Malays. Math. Sci. Soc. 39 (2016), 839-848.

[5] I. Banič and M. Črepnjak, Markov pairs, quasi Markov functions and inverse limits, Houston J. Math. 44 (2018), 695-707.

[6] M. Črepnjak and T. Lunder, Inverse limits with countably Markov interval functions, Glas. Mat. Ser. III 51(71) (2016), 491-501.

[7] L. Alvin and J. P. Kelly, Markov set-valued functions and their inverse limits, Topology Appl. 241 (2018), 102-114.

[8] W. T. Ingram, Inverse limits of upper semi-continuous functions that are unions of mappings, Topology Proc. 34 (2009), 17-26.

T. Sovič

Faculty of Civil Engineering, Transportation Engineering and Architecture

University of Maribor

2000 Maribor

Slovenia

E-mail: tina.sovic@um.si

Received: 2.8.2018.

Revised: 22.1.2019. 